\title{
Shared Psychotic Disorder, A Case Report and Review of the Concept 'Folie A Famille'
}

\section{Amauri Clozer Pinheiro and Silvia Marli Magrin Saullo*}

Rua José Zorzenon 222, Ribeirania - Ribeirão Preto - SP, Brazil

*Corresponding Author: Silvia Marli Magrin Saullo, Rua José Zorzenon 222,

Ribeirania - Ribeirão Preto - SP, Brazil.
Received: July 03, 2021

Published: August 05, 2021

(C) All rights are reserved by Amauri Clozer

Pinheiro and Silvia Marli Magrin Saullo.

\begin{abstract}
It is an unusual mental disorder known as Shared Psychotic Disorder characterized by sharing an illusion between two or more people who are in a close relationship. The primary individual, who has a psychotic disorder with delusions, influences another individual or more with a specific belief. Usually found between two individuals, but it can include larger groups, or even the family, being called 'Folie a Famille'. We bring the case of a man who died and his family did not accept the autopsy, claiming that the individual was alive, even with all the evidences pointing the opposite.
\end{abstract}

Keywords: Death; Shared Psychotic Disorder; "Folie a Famille"

\section{Introduction}

The first French neurologist and psychiatrist to report this condition in 1860 was Jules Gabriel François Baillarger, who also used the term ‘comunicado folie' (communicated psychosis). In German psychiatry, it was called 'Induzierts Irresein' by Lehman and Sharfetter [1].

In 1877, Lasegue and Falret published the article entitled 'Madness for two' presenting it as separate nosographic entity [1].

The French word 'Folie a deux' means madness shared by two. The concept highlighted the delusional idea could be shared between two closely connected people or even more. Alexander Gralnick in his review of 103 'Folie a deux' cases, described four types of this disorder. He defined the disorder as being characterized by the transparency of delusions and/or abnormal behavior from one person to another (or several others) that have a close relationship with the principal one affected.

Modern classifications adopt the name of Shared Psychotic Disorder in both ICD 10 (International Classification of Diseases) and DSM IV (Diagnostic and Statistical Manual of Mental Disorders).
The issue of the nosographic framework is still relevant, which translates into the difficulty of bringing objectives criterias to a concept that is often confusing. We prefer not to delve into these points, as they are not the objectives of our report.

\section{Objective and Method}

It aims to pay attention to the existence of unusual behavior in the light of science using simple observation as a method correlating it to the cases of shared psychotic disorders described in the literature.

\section{Case Report}

The death verification service (SVO) received an adult body, 84 years old, with a death record due to natural death sent from the UPA (Emergency Care Unit) indicating death due to probable acute cardiac etiology after receiving first aid at the unit. The referral guide indicated the death that occurred at 6:43 p.m., Saturday, at the beginning of the year of 2020 and along with it there was also a police report and the request for the necroscopic examination required by the police authority to determine the cause of death. 
On Sunday morning the wife and daughters who desperately asked for an autopsy not to be performed because they believed their relative 'was still alive'attended. Crying they said that among their family members a similar case had already occurred with an apparently dead person and that 'they knew that this was happening to their relative and they needed to prevent the act', having already hired a lawyer to intervene with the police chief.

More than 16 hours after the initial death reports we provided the entrance of the wife and daughters for visual identification of the corpse and as they approached the body, they were upset, very agitated, with uncontrolled crying, screaming, mentioning that 'they knew he was still alive and that they could feel it in their thoughts'. They did this in a desperate and uncontrolled way, even in the face of the technical evidence indicated, such as: no breath, dorsal hypostasis, dehydration, skin pallor and cadaveric stiffness. Even though, they shouted claiming that the 'body was still alive and that he could hear them'.

Maintaining this behavior, they left without convicing and after the reiteration of the exam by the police authority we were surprised by the appearance of doctors from SAMU (Mobile Emergency Care Service) who had been requested by the family. In an attempt to calm the spirits, and allow necropsy to begin, the doctors present there opted to perform an ECG (Eletrocardiogram) that was shown to the family, confirming once again the death. But even so, for not being able to convince the medical team of the "existence of life" in the corpse, they remained unhappy waiting for the completion of the technical procedure.

After the evaluation, death was found to be of cardiac origin and a declaration was provided that allowed the removal of the body and the transfer to the funeral.

\section{Discussion}

A review found that $98 \%$ of cases reported between 1993 and 2005 occurred within the same family, usually between married couples $(52 \%)$ or sisters (24\%). In addition, about $50 \%$ of cases are mother/daughter or sister/sister couples. According to Alexander Gralnick, about 8\% of a series of cases occurs among patients or even non-consanguineous friends [2].

Scharfetter stated that the age range of the inducing partner is from 16 to 82 years old (average 38.5 years) and that the induced partner (submissive) is from 4 to 79 years old (average 36.2 years). There was also a report that the submissive individual is charac- teristically younger, less intelligent, more naive and more passive, with lower self-esteem than the inducer, although these findings have not been consistently replicated [2].

The disorder usually begins with a difference in power and influence between the two, where the one who induces psychotic symptoms is the 'real'psychotic, usually an energetic and active individual [2].

Environmental factors also play an important etiological role in causality. The very definition of the condition denotes an intimate relationship over the years and a common sharing of illusions, as well as those who live in increasing isolation tend to become more suspicious and threatened, which can lead to the production of paranoid reactions [3].

Death has a great importance in the sense of our existence, because we know we are mortal, but we don't know the moment of our death. As a result, we live as if we are immortal knowing we are not. Any approach to death determines substantial changes in the direction of our lives. Our fear of dying is always present so that a part of our vital energy is consumed in the task of denying death.

Under the law, the physician must sign a death certificate for the purpose of confirm the existence of death and register its cause, from a medical point of view and possible legal applications, thus allowing the diagnosis of the legal cause of death [4].

But often the simplest and most obvious things are the hardest to conceptualize and define, not to mention the understanding and acceptance that goes through historical social, anthropological, cultural, religious phenomena etc...

Death is no longer an easy concept to build. Affirming that an individual is alive or dead depends on the understanding that one has of the concept of death and this can vary according to different cultures, religions or scientific approaches. The medical and legal definition of death changed less than 30 years ago, from cardiorespiratory arrest to the so-called neurological death. In addition, numerous protocols are periodically revised, expanding this discussion to ensure the concepts [4].

The biological limit between life and death may be precise, but from a psychological point of view both fuse. Freud wrote several articles on death and maintained that we have within us an instinct for life (EROS) and an instinct for death (Thanatos). We are always trying to dispense death, eliminate it from life. For this we use tools 
such as silence (not talking about it), medications, addictions, religion and sometimes we get lost in pathology, in an eagerness to deny one`s finitude [4].

In medical practice, the detection of death is characterized by the presence of immediate abiotic signs and mediate abiotic signs, also called consecutive [5]. The first one are verified by the verification of cardiac and respiratory arrest, loss of consciousness, disappearance of movements and muscle tone, loss of reflex action to tactile, thermal and painful stimuli, sphincter relaxation, absence of pulse, partially closed eyelids, hippocratic facies and loss of brain function. The consecutive abiotic phenomena, are those that will establish themselves over time due to the arrest of the metabolic function and they are characterized by the gradual cooling of the body, cadaveric stiffness, cadaveric spasm, hypostasis spots and dehydration [6]. In the present case, the criteria for immediate death and consecutive death were met.

In relation to the facts narrated, perhaps it was a sum of things that we don't know. The components of the equation that led to this close and fusional relationship, with a total identification with a psychotic content, may have led to the behavior displayed by this family.

The observed behavior suggests that one of the family members was the inducer, due to the presence of disorders of the judgement with delusional disorder and similar behavior of denying death, despite the reality data presented. During the episode we observed the strangeness in the behavior: "...they knew he was still alive and could feel it in their thoughts", "... they whispered next to the body indicating that the corpse could hear them and asked him to wake up".

We consider that this pathology responds to a multifactorial natures, including psychological, sociological, cultural, religious and even genetical reasons and deserves further scientific studies. It is worth pointing out the research difficulties, considering that the case reported occurred in a short period, in which the intervention of public bodies for legal and sanitary purposes was necessary.

\section{Conclusion}

Through this case report we seek to propose reflections on shared denial of death, in a family nucleus, as well as the psychopathology of delusional disorders, in the case of 'Folie a Famille'. We brought an experience that transcends the limits of our specialities, thus demonstrating the need for continuous study of the various areas of science in addressing the theme.

\section{Conflicts of Interest}

The authors declare that they have no conflict of interest.

\section{Funding Source}

None.

\section{Authors` Participation}

- $\quad$ Clozer Pinheiro, A: Data collect, necropsy performance.

- Saullo Magrin, SM: Bibliographic Research and Analysis.

- Clozer Pinheiro, A and Saullo Magrin, SM: Reading, discussion, joint writing of content and final writing.

- Saullo Magrin, SM: Article submission.

\section{Bibliography}

1. Feras Al Saif and Yasir Al Khalili. Shared Psychotic Disorder PubMed.gov - StatPearls Publishing (2021).

2. Gabrieli Cipriani., et al. "A Contagious Disorder: Folie à Deux and Dementia". American Journal of Alzheimer's Disease and Other Dementiasin (2018).

3. Suresh Kumar P N., et al. "Folie a deux". Indian Journal of Psychiatry 47 (2005):164-166.

4. Santos MCCL. dos. "Conceito médico-forense de morte". Revista Da Faculdade De Direito, Universidade De São Paulo, 92 (1997): 341-380.

5. FÁVERO Flamínio. Medicina Legal: Introdução ao estudo da Medicina Legal, Identidade, Traumatologia. 11a ed., 1966 Belo Horizonte, Itatiaia 25.1 (1975): 532-554.

6. Souza Paula Helena dos Santos., et al. "A Tanatognose por observação dos Fenômenos Cadavéricos". Revista Científica Multidisciplinar Núcleo do Conhecimento. Ano 03, Ed.07 06 (2008): 28-42.

\section{Volume 4 Issue 9 September 2021 (c) All rights are reserved by Amauri Clozer Pinheiro and Silvia Marli Magrin Saullo.}

